\title{
UTILIZAÇÃO DO INSTRUMENTO DE MELHORIA DE DESEMPENHO NAAVALIAÇÃO DO EXAME DE PAPANICOLAU POR ENFERMEIROS
}

\author{
Kylvia Gardênia Torres Eduardo¹, Escolástica Rejane Moura Ferreira², Ana Karina Bezerra Pinheiro³, Lorena Barbosa
}

Ximenes $^{4}$

\begin{abstract}
RESUMO: O estudo teve por objetivo avaliar o desempenho de enfermeiros do Programa Saúde da Família (PSF) quanto à realização do exame de prevenção do câncer de colo uterino, tomando por base o Instrumento de Melhoria do Desempenho (IMD), da Secretaria da Saúde do Ceará. Realizou-se no município de Paraipaba-CE, em setembro de 2005, com os sete enfermeiros do Programa. Utilizou-se a técnica da observação estruturada direta durante a execução de 21 exames. As participantes alcançaram, parcialmente, o padrão de qualidade estabelecido no IMD, devido à deficiência na infra-estrutura do serviço, escassez de recursos materiais e capacitação técnica insuficiente. Constatou-se, pois, a necessidade de trabalhar essas lacunas, planejando ações que as amenizem ou solucionem. O IMD mostrou-se eficaz como instrumento para avaliação, indicando caminhos para a melhoria da qualidade do serviço.

PALAVRAS-CHAVE: Esfregaço vaginal; Qualidade de assistência à saúde; Colo do útero; Neoplasias; Prevenção.
\end{abstract}

\section{USE OF THE PERFORMANCE IMPROVEMENT INSTRUMENT IN THE EVALUATION OF PAP SMEARS CARRIED OUT BY NURSES}

\begin{abstract}
The study objectified to evaluate nurses' performance from Family Health Program in the preventive cervical cancer exam by means of the Performance Improvement Instrument (PII), of Cearás Health Secretary. It was carried out in the municipality of Paraipaba - Ceará State/ Brazil in September of 2005, by seven nurses from the Program. Data had been collected through the technique of direct structured observation of 21 exams. The participants had partially reached the quality standards established in the PII due to deficiencies in the service infra-structure, supply scarcity, and poor technical qualification. Therefore, it was evidenced the need to fill in these gaps, planning actions in order to remediate or solve them. The PII showed efficient as an evaluation instrument, pointing to ways for the improvement of the service quality.
\end{abstract}

KEYWORDS: Pap smears; Quality of health care; Cervix uteri; Neoplasms; Prevention.

\section{USO DEL INSTRUMENTO DE MEJORÍA DEL FUNCIONAMIENTO EN LA EVALUACIÓN DE FROTIS VAGINAL EJECUTADOS POR ENFERMERAS}

\begin{abstract}
RESUMEN: El estudio tuvo como objetivo evaluar la examinación de la prevención de los neoplasmas cervicales uterinos ejecutada por las enfermeras de Programa de Salud de la Familia (PSF) tomando por base el Instrumento de Mejoría de Desempenho (IMD), de la Secretaría de Salud de Ceará. Fue realizado en Paraipaba, CE, en septiembre de 2005, con siete enfermeros del Programa. Se ha utilizado la técnica da observación estructurada directa durante la ejecución de 21 exámenes. Las participantes llegaron, parcialmente, al estándar de cualidad establecido en IMD, a causa de la deficiencia en infraestructura del servicio, escasez de recursos materiales y capacitación técnica insuficiente. Se ha constatado, pues, la necesidad de trabajar tales situaciones, planeando acciones que las amenizen o solucionen. El IMD se ha mostrado eficaz como instrumento para evaluación, indicando caminos para la mejoría de cualidad del servicio.
\end{abstract}

PALABRAS CLAVE: Frotis vaginal; Cualidad de la atención de salud; Cuello del útero; Neoplasias; Prevención.

${ }^{1}$ Enfermeira. Mestre em Enfermagem. Programa Saúde da Família de Paraipaba-CE.

${ }^{2}$ Enfermeira. Doutora em Enfermagem. Professora Adjunta II da Universidade Federal do Ceará-UFC.

${ }^{3}$ Enfermeira. Doutora em Enfermagem. Professora Adjunta II da UFC. Co-Tutora do Programa de Educação Tutorial- PET-Enfermagem/UFC.

${ }^{4}$ Enfermeira. Doutora em Enfermagem. Professora Adjunta III da UFC. Tutora do Programa de Educação Tutorial- PET-Enfermagem/ UFC

Autor correspondente:

Escolástica Rejane Ferreira Moura

Av. Filomeno Gomes, 80 - 60010-280 - Fortaleza-CE Recebido: 01/06/08

E-mail:escolpaz@yahoo.com.br

Aprovado: 25/09/08 


\section{INTRODUÇÃO}

O câncer de colo uterino (CCU) é uma neoplasia maligna que, apesar de apresentar decréscimo no índice de mortalidade nos últimos anos, persiste com cifras elevadas. É considerado o segundo tipo de câncer mais comum na população feminina, perdendo somente para o câncer de mama, sendo responsável pela morte de 230.000 mulheres a cada ano. Estima-se que no Brasil, em 2008, o número de casos novos será de 18.680 , com um risco estimado de 19 casos a cada 100 mil mulheres ${ }^{(1)}$.

Este tipo de câncer se inicia com uma lesão pré-invasiva, curável em até $100 \%$ dos casos detectados precocemente. O Papiloma Vírus Humano (HPV), o Herpesvírus tipo II (HVS), tabagismo, início precoce de atividade sexual (antes dos 18 anos), pluralidade de parceiros sexuais, baixas condições sócio-econômicas e subnutrição constituem-se fatores de risco importantes para o desenvolvimento das alterações epiteliais, podendo evoluir, no decorrer de vários anos, para um câncer invasor ${ }^{(2)}$.

Conquanto se constitua uma condição maligna, o CCU é uma das patologias com maior possibilidade de diagnóstico precoce, o qual é fornecido pela citologia do esfregaço cérvico-vaginal ou exame de Papanicolaou, considerado o método de rastreamento universal para câncer de colo uterino ${ }^{(3)}$.

A coleta citológica ou exame de Papanicolaou é realizado, no Brasil, na rede básica de saúde, por médicos ou enfermeiros devidamente capacitados. A coleta realizada por enfermeiros é respaldada na Portaria Técnica da SAS/MS nº.1230 de 14/10/1999 que estabelece as categorias profissionais habilitadas para essa prática ${ }^{(4)}$. No cotidiano das equipes do Programa Saúde da Família (PSF), o enfermeiro está engajado em todas as atividades de controle do CCU, sendo relevante seu papel nas mesmas. Tem-se, inclusive, que o maior número de coletas citológicas na atenção primária é realizado por enfermeiros ${ }^{(5)}$.

O Instituto Nacional de Câncer reforça a efetividade do exame, pelo fato deste apresentar redução das taxas de incidência de câncer invasor em até $90 \%$ quando o rastreamento apresenta boa cobertura e é realizado dentro dos padrões de qualidade ${ }^{(6)}$.

Na perspectiva de fornecer um atendimento de qualidade, a Secretaria de Saúde do Ceará (SESACE) preconiza etapas e técnicas que devem ser realizadas durante o atendimento em prevenção do CCU. Em parceria com três agências de cooperação internacional (Program for International Education in Reproductive Health (JHPIEGO), Management Sciences for Health (MSH) e Center for Communication on Programs (CCP)) a SESA desenvolveu, de 1996 a 2000, um Programa de Qualidade (PROQUALI), estabelecendo um conjunto de padrões ou protocolos, denominados Instrumentos de Melhoria de Desempenho (IMD), a ser adotado para o alcance da qualidade das ações prestadas pela equipe de PSF, tendo como pressupostos básicos a auto-avaliação e a autogestão.

O IMD pode ser aplicado de forma sistemática a fim de promover auto-aprendizagem, auto-avaliação e avaliação externa ${ }^{(7)}$. Portanto, a qualidade do atendimento do enfermeiro na prevenção do CCU pode ser avaliada a partir da verificação do cumprimento ou não dos padrões estabelecidos pelo PROQUALI. Essa avaliação possibilita um processo reflexivo da prática realizada e gera, conseqüentemente, transformação da realidade e crescimento dos sujeitos envolvidos ${ }^{(8)}$.

Levando-se em consideração que a redução das taxas de incidência do CCU dependem da qualidade da coleta citológica, que o enfermeiro é o principal responsável pela coleta na atenção básica e que o PROQUALI disponibiliza um instrumento para a avaliação destes profissionais na prevenção do CCU, questionou-se: os enfermeiros estão realizando o exame de prevenção do CCU, segundo os padrões do IMD?

Assim, verificou-se a relevância de realizar este estudo a fim de obter dados relativos à situação atual de realização do exame de prevenção do CCU por enfermeiros, estabelecendo uma linha de base para posteriores avaliações, bem como apresentar parâmetros para promover melhoria da assistência.

Este estudo tem como objetivo avaliar a qualidade do exame de prevenção do câncer de colo uterino realizado por enfermeiros do PSF, tendo como padrão de referência de qualidade o instrumento elaborado pelo PROQUALI.

\section{MATERIAL E MÉTODO}

Estudo descritivo-exploratório, desenvolvido com enfermeiros do PSF do município de ParaipabaCE. O PSF, neste município, conta com sete equipes em todo o seu território, o que corresponde a sete enfermeiros inseridos no Programa, atuando na área de prevenção do CCU e, portanto, realizando a coleta de material para o exame de Papanicolaou.

Foram selecionados os sete enfermeiros do PSF

Cogitare Enferm 2008 Jul/Set; 13(3):329-35 
do município por se enquadrarem nos seguintes critérios de seleção: ser enfermeiro, integrar o Programa Saúde da Família e realizar o exame de prevenção do CCU.

Os dados foram coletados com a utilização do instrumento de melhoria do desempenho (IMD) do Projeto PROQUALI, aplicado através da observação sistemática e direta dos enfermeiros, nos seus respectivos campos de trabalho (Unidades Básicas de Saúde). O IMD dirigido para a prevenção do CCU é composto por 20 procedimentos que descrevem o atendimento, passo a passo. Como parâmetro de avaliação, o PROQUALI sugere que quando o procedimento for realizado adequadamente o mesmo seja categorizado como SIM; quando não for realizado, ou for inadequadamente, seja categorizado como NÃO; e quando a situação específica de um atendimento não se fizer necessária à realização de um determinado passo, este seja categorizado como "Não se aplica”(9).

De acordo com o PROQUALI, ao se utilizar o IMD para efeito de avaliação, o procedimento deverá ser observado, no mínimo, em três situações, com a finalidade de evitar falsas avaliações por situações “maquiadas” pelos participantes. Esse aspecto é corroborado por outros autores que orientam para a mesma estratégia, ressaltando o princípio da “reatividade”, resposta do sujeito observado, que é capaz de alterar os resultados reais de um estudo ${ }^{(10)}$.

Os dados foram coletados no mês de setembro de 2005. Primeiramente, foi realizado o mapeamento das equipes de PSF do município, verificando o dia da semana que cada equipe reservava para realizar atendimento de prevenção de câncer de colo uterino. Na seqüência, os enfermeiros foram contactados e esclarecidos quanto à pesquisa, o período de coleta e seus direitos como participantes. Foram realizadas visitas semanais, sendo observados três atendimentos por enfermeiro, totalizando 21 atendimentos observados.

O IMD apresenta o atendimento para prevenção do CCU em três etapas: acolhimento, técnica do exame e seguimento/encaminhamento da usuária. Todavia, este estudo se restringiu à segunda etapa, a técnica do exame propriamente dito, o que corresponde a 15 itens do IMD.

O projeto foi autorizado pelo Comitê de Ética em Pesquisa da Universidade Federal do Ceará, segundo o protocolo $n^{\circ}$ 237/05. As participantes assinaram o Termo de Consentimento Livre e Esclarecido, bem como as clientes submetidas ao exame, após orientação sobre o estudo e permissão destas para a observação do atendimento, seguindo a
Resolução 196/96 do Conselho Nacional de Saúde, Ministério da Saúde que trata de pesquisa com seres humanos $^{(11)}$.

Os dados foram organizados em planilha eletrônica (EXCEL) e analisados de acordo com a literatura pertinente.

\section{RESULTADOS E DISCUSSÃO}

A Tabela 1 mostra que, dos 315 (100\%) itens observados, 188 (59,7\%) foram realizados adequadamente, 104 (33,0\%) não foram realizados e 23 (7,3\%) não se aplicaram às situações observadas. Portanto, a maioria dos procedimentos foi realizada, mas porcentagem significativa deixou de ser executada satisfatoriamente.

Ao investigar fatores de risco, tem-se a possibilidade de se detectar DSTs, dentre elas o HPV, grande potencializador de alterações celulares, bem como de realizar atividade educativa, fornecendo informações à cliente de forma que esta perceba os riscos, as mudanças do corpo e passe a adotar medidas preventivas adequadas ${ }^{(12)}$.

Nos 21(100\%) procedimentos relacionados à investigação de fatores de risco, 15 (71,4\%) foram realizados. Isso ocorreu pelo fato de as profissionais terem se limitado aos fatores que constam no prontuário ou no formulário do Sistema de Informação do Câncer do Colo do Útero (SISCOLO).

A importância de conhecer não somente os fatores de risco, mas também os aspectos sócioeconômicos relacionados com o CCU, fornece subsídios para o planejamento das ações de controle da neoplasia ${ }^{(13)}$.

A lavagem das mãos com água e sabão, antes e após o exame, e a secagem com toalha de papel é medida de biossegurança necessária. Esse procedimento é considerado o mais importante para prevenção da transmissão de infecção. A sua realização é exigida em procedimentos em que ocorra contato prolongado e intenso com o paciente, antes e após a remoção de luvas de procedimento e/ou quando há contato com mucosas, sangue, fluidos corporais, secreções e excreções, bem como o contato com fômites que provavelmente estejam contaminados ${ }^{(14-15)}$. A secagem das mãos deve ser feita com toalha de papel, pois as toalhas convencionais permanecem úmidas, possibilitando a transmissão de microorganismos. O papel toalha deve ser mantido sempre no suporte e adquirido em toalhas individuais e não em rolo ${ }^{(2)}$. 
Tabela 1. Distribuição dos procedimentos do exame de prevenção do câncer de colo uterino segundo desempenho dos enfermeiros do PSF. Paraipaba-CE, 2006

\begin{tabular}{|c|c|c|c|c|c|c|c|c|}
\hline \multirow{2}{*}{ Prevenção do CCU passo a passo } & \multicolumn{2}{|c|}{$\operatorname{Sim}(S)$} & \multicolumn{2}{|c|}{ Não(N) } & \multicolumn{2}{|c|}{$\begin{array}{l}\text { NA (Não } \\
\text { se aplica) }\end{array}$} & \multicolumn{2}{|c|}{ Total } \\
\hline & $\mathrm{n}$ & $\%$ & $\mathrm{n}$ & $\%$ & $\mathrm{n}$ & $\%$ & $\mathrm{~N}$ & $\%$ \\
\hline $\begin{array}{l}\text { 1. Investiga fatores de risco (multiplicidade de parceiros, DST de } \\
\text { repetição, início precoce da atividade sexual). }\end{array}$ & 15 & 71,4 & 6 & 28,6 & --- & --- & 21 & 100 \\
\hline 2. Lava as mãos com água e sabão e enxuga-as com toalha de papel. & --- & --- & 21 & 100 & --- & --- & 21 & 100 \\
\hline 3. Coloca as luvas de procedimento nas duas mãos. & 21 & 100 & --- & --- & --- & --- & 21 & 100 \\
\hline 4. Examina o espéculo adequadamente, sem lubrificar. & 18 & 85,7 & 3 & 14,3 & --- & --- & 21 & 100 \\
\hline 5. Realiza a abordagem sindrômica para DST conforme o fluxograma. & 0 & 0 & 0 & 0 & 21 & 100 & 21 & 100 \\
\hline $\begin{array}{l}\text { 6. Coleta material da ectocérvice com a extremidade denteada da } \\
\text { espátula de Ayre (coloca a extremidade maior no orifício do colo, } \\
\left.\text { fixa e gira } 360^{\circ}\right) \text {. }\end{array}$ & 20 & 95,2 & --- & --- & 1 & 4,8 & 21 & 100 \\
\hline $\begin{array}{l}\text { 7. Coleta material no canal cervical com escovinha, fazendo uma } \\
\text { rotação de } 180^{\circ} \text { (exceto em gestantes). }\end{array}$ & 11 & 52,4 & 9 & 42,9 & 1 & 4,8 & 21 & 100 \\
\hline $\begin{array}{l}\text { 8. Coloca o material coletado na lâmina previamente identificada, } \\
\text { colocando a espátula paralelamente à lâmina e arrastando-a } \\
\text { suavemente no longitudinal, sem batê-la. }\end{array}$ & 21 & 100 & --- & --- & --- & --- & 21 & 100 \\
\hline $\begin{array}{l}\text { 9. Coloca a lâmina imediatamente no álcool a mais de } 90 \% \text {, de } \\
\text { maneira que fique totalmente imersa. }\end{array}$ & 17 & 81,0 & 4 & 19,0 & --- & --- & 21 & 100 \\
\hline $\begin{array}{l}\text { 10. Realiza técnica de inspeção cervical com ácido acético a } 5 \% \text { para } \\
\text { pesquisar lesões acetobrancas. Espera } 1 \text { minuto após a colocação do } \\
\text { ácido para realizar a leitura. Em caso positivo, realizar e/ou encaminhar } \\
\text { para colposcopia. }\end{array}$ & 2 & 9,5 & 19 & 90,5 & --- & --- & 21 & 100 \\
\hline $\begin{array}{l}\text { 11. Realiza teste de Schiller com solução de Lugol para evidenciar } \\
\text { ectopias. }\end{array}$ & 21 & 100 & --- & --- & --- & --- & 21 & 100 \\
\hline $\begin{array}{l}\text { 12. Retira o espéculo com cuidado para não prender o colo entre as } \\
\text { lâminas do espéculo; não retirá-lo aberto, pois pode causar dor e } \\
\text { traumatismo uretral. }\end{array}$ & 21 & 100 & --- & --- & --- & --- & 21 & 100 \\
\hline 13. Coloca o instrumental usado no balde para material contaminado. & 18 & 85,7 & 3 & 14,3 & --- & --- & 21 & 100 \\
\hline $\begin{array}{l}\text { 14. Despreza as luvas e os materiais contaminados em recipiente com } \\
\text { saco plástico identificado para material contaminado. }\end{array}$ & --- & --- & 21 & 100 & --- & --- & 21 & 100 \\
\hline 15. Lava as mãos com água e sabão e enxuga-as com toalha de papel. & 3 & 14,3 & 18 & 85,7 & --- & --- & 21 & 100 \\
\hline TOTAL & 188 & 59,7 & 104 & 33,0 & 23 & 7,3 & 315 & 100 \\
\hline
\end{tabular}

Fonte: Instrumento de Melhoria do Desempenho (IMD), SESA-CE.

Para o procedimento "lavagem das mãos antes da coleta” verificou-se que este não foi realizado nos $21(100 \%)$ procedimentos observados. Em relação à lavagem das mãos após a coleta e retirada das luvas, constatou-se a realização deste somente em $3(14,3 \%)$ dos 21 exames observados. Entretanto, é importante enfatizar que, após a remoção das luvas, as mãos foram lavadas, porém não foram secas com toalha de papel, e sim com toalhas de tecido que, segundo as enfermeiras, eram trocadas a cada turno. Todavia, na ausência de toalha de papel, aconselha-se secar as mãos com toalha individual, lavada diariamente ${ }^{(15)}$.

Ressalta-se, ainda, que em algumas unidades as toalhas de papel estavam disponíveis, porém não foram utilizadas, segundo as participantes, ou por não ser hábito da profissional ou por falta de suporte. Em somente uma unidade, a não realização da lavagem das mãos se deu pela ausência de lavatório no consultório. 
Alguns dos 19 exames em que o procedimento "lavagem das mãos após a coleta” foi considerado como NÃO realizado, foi classificado nessa categoria por ter sido realizado de forma inadequada devido à não utilização de sabão, mesmo estando disponível. A higiene das mãos talvez ganhasse maior adesão se as enfermeiras tivessem à disposição solução de álcool glicerinado como opção(14).

Com relação às medidas de segurança, observou-se que nos 21 (100\%) exames as luvas foram postas nas duas mãos. Como na lavagem das mãos, as luvas devem ser usadas quando há contato com membranas, mucosas e/ou pele não íntegra. Deve ser utilizado um par de luvas diferente para cada cliente, a fim de evitar contaminação cruzada. O uso de luvas não elimina a necessidade de lavar as mãos ${ }^{(15)}$.

Quanto ao espéculo ser introduzido sem lubrificante, constatou-se a sua realização em 18 (85,7\%) exames. O espéculo não deve ser introduzido lubrificado, porque os lubrificantes comerciais interferem com os achados da citologia cervical ${ }^{(16)}$. No entanto, orienta-se que os espéculos sejam lubrificados em água morna antes de serem introduzidos, facilitando o exame e promovendo relaxamento da musculatura pélvica ${ }^{(17)}$. Tal prática pareceu inviável nas unidades de saúde pesquisadas, já que estas não dispõem de torneiras com aquecimento elétrico.

Observou-se uso de ácido acético como lubrificante em $3(14,3 \%)$ exames, a fim de facilitar sua colocação e promover conforto, o que é desaconselhado.

A abordagem sindrômica, instrumento que utiliza fluxogramas desenvolvidos e testados para diagnóstico precoce e tratamento imediato das DST, não foi aplicada nos 21 (100\%) exames observados, pois as profissionais não estavam legalmente respaldadas no município para prescrever as medicações preconizadas na respectiva estratégia de atendimento ${ }^{(12)}$. Mesmo assim, não há justificativa para omitir a inspeção e investigação de sinais e sintomas de DST, seguidas de encaminhamento para o médico. Por outro lado, é lamentável que os enfermeiros, no decorrer do exame de prevenção, tenham a oportunidade perdida para detectar e tratar a maioria das DSTs, comprometendo a saúde e a vida de milhares de mulheres por falta de definição legal para o exercício profissional. Autores enfatizam que através da abordagem sindrômica o enfermeiro poderia não só implementar o tratamento precocemente, como também reduzir a transmissibilidade e oferecer informações para a prática sexual segura e a quebra da cadeia de transmissão, bem como rastrear a sífilis e o $\mathrm{HIV}^{(5)}$.
A coleta do material citológico é realizada com espátula de Ayre que traz células da ectocérvice. Devese colocar a extremidade mais longa da espátula no óstio do colo, girando $360^{\circ}$, tendo o cuidado de incluir a zona de transformação e a junção escamocolunar (JEC), quando visualizada. Aconselha-se realizar primeiro essa coleta para evitar contaminação da lâmina com sangue, que às vezes aparece após o uso da escovinha ${ }^{(17)}$.

Observou-se, então, a coleta com espátula em $20(95,2 \%)$ procedimentos e $1(4,8 \%)$ não se aplicou (NA), devido à cliente ser histerectomizada.

O material da endocérvice é coletado com escovinha, girando-a $180^{\circ}$. O objetivo de utilizar a escovinha deve-se ao fato da junção escamocolunar (JEC) estar alta no canal cervical em algumas ocasiões $^{(14)}$. O Ministério da Saúde ressalta que a escovinha só deve ser utilizada quando a JEC não for visualizada e deve ser evitada em gestantes ${ }^{(18)}$.

A coleta com escovinha foi realizada em 11 (52,4\%) dos 21 exames. Um dos procedimentos não se aplicou (NA) pelo motivo já descrito anteriormente. Constatou-se, ainda, a coleta do conteúdo de fundo de saco, fixado na lâmina juntamente com os materiais da ectocérvice e endocérvice.

A baixa coleta com escovinha teve como causa, segundo as participantes, a falta de insumo. Entretanto, dos 9 (42,9\%) exames nos quais não foram coletados material com escovinha, a não realização dessa prática poderia ter sido classificada como NA, pois algumas clientes apresentaram JEC visível, permitindo que esse material fosse colhido também com a espátula. Contudo, em três procedimentos a enfermeira não coletou células com escovinha, mesmo tendo disponibilidade de material, argumentando que a clientela poderia omitir informação sobre gravidez, na qual o procedimento é contra-indicado. Esta preocupação deve ser superada através de uma anamnese bem conduzida, evitando conduta incorreta uma vez que uma coleta adequada exige a presença de células endocervicais.

Após a coleta, o material deve ser colocado na lâmina previamente identificada com as iniciais do nome da cliente e o número do prontuário. $\mathrm{O}$ registro deve ser feito com lápis, pois a tinta de uma caneta, por exemplo, pode borrar, prejudicando a leitura da identificação ${ }^{(16)}$. O material colhido deve ser aplicado em uma mesma lâmina, em fina espessura, de forma suave, regular e rápida. A fixação é realizada com álcool a $95 \%$ ou spray tipo carbovax, imediatamente após a aplicação, visando a conservação das características celulares. Quando fixada com álcool, 
a lâmina deve ficar totalmente imersa ${ }^{(2)}$.

A "colocação do material na lâmina" e sua "fixação" foram realizados adequadamente em 21 $(100 \%)$ e 17 (81\%) dos procedimentos, respectivamente. O álcool utilizado para a fixação era o absoluto, seguindo as exigências do IMD, o qual estabelece a porcentagem do álcool acima de $90 \%$. Entretanto, o motivo de 4 dos procedimentos relativos à fixação do material na lâmina serem classificados como não realizados se deu pela imersão incompleta das lâminas no álcool, como recomendam a literatura e o IMD.

Após a coleta, a técnica segue com a inspeção do colo uterino utilizando ácido acético e lugol. Primeiro, realiza-se a inspeção visual com ácido acético (IVA). Para tanto, o colo e o fundo de saco vaginal devem ser limpos com algodão ou gaze seca, pincelados com o ácido acético a $5 \%$, esperando um a dois minutos para a leitura. O ácido acético a 5\% coagula as proteínas tornando esbranquiçadas as lesões causadas pelo Papiloma Vírus Humano (HPV).

A inspeção com lugol, também chamada teste de Schiller, permite diferenciar as alterações da mucosa do colo, devido às células terem a propriedade de se impregnar com iodo, corando-se em grau variado de acordo com o teor de glicogênio que contêm. Portanto, células com alterações se impregnam menos que as células normais, apresentando resultado compatível com Schiller positivo ${ }^{(2)}$.

A inspeção do colo com ácido acético foi realizada em somente 2 (9,5\%) exames. Esse resultado deve-se ao fato de o ácido acético utilizado estar em concentração inadequada (2\%). Em pouco mais da metade dos exames observados, verificou-se que as enfermeiras não esperavam tempo adequado para leitura, fazendo-o apenas em casos sugestivos de alterações, detectados na anamnese.

O teste de Schiller foi realizado em 21 (100\%) dos exames observados, portanto, satisfazendo a recomendação do IMD.

O espéculo deve ser retirado lentamente, ficando aberto até que o colo fique livre. A seguir é empurrado para baixo, sendo aplicada pressão na parede posterior da vagina, permitindo o fechamento à medida que é retirado, evitando tanto a distensão excessiva quanto o pinçamento da mucosa ${ }^{(19)}$. Observou-se em todos os exames a retirada adequada do espéculo.

O material contaminado reprocessável (instrumental) foi descartado em balde identificado para material contaminado em 18 (85,7\%) dos procedimentos.
Contudo, o lixo comum não foi desprezado em saco plástico identificado. $O$ instrumental utilizado no exame era colocado, em sua maioria, em balde contendo água, hipoclorito de sódio e sabão, para posterior limpeza e esterilização, quer em estufa ou autoclave. Em 3 procedimentos, a profissional não dispôs de balde exclusivo para colocar o instrumental, desprezando-o no cesto juntamente com o lixo contaminado.

Quanto ao material contaminado, apesar de ser desprezado em cesto com saco plástico, o procedimento obteve baixa porcentagem de realização, devido ao saco não ser identificado para lixo contaminado. Todo lixo contaminado era queimado na própria unidade ou no hospital municipal, segundo as participantes.

A qualidade da técnica ficou comprometida, algumas vezes, pela escassez de recursos, como por exemplo, a falta de escovinha, papel toalha, sabão e ácido acético a 5\%; problemas de infra-estrutura, como ausência de lavatório, suporte para papel toalha e recipiente para colocação do material contaminado no local onde se realiza o exame.

\section{CONCLUSÃO}

O IMD voltado à prevenção do CCU fornece as diretrizes para um atendimento de elevado padrão técnico. Esse instrumento, além de fornecer o padrão de excelência da técnica a ser realizada, também pode ser aplicado como parâmetro para avaliar o serviço prestado, apontando os avanços e as deficiências ou lacunas encontradas no mesmo. Nesse contexto, podem-se planejar ações que amenizem ou solucionem as deficiências encontradas, possibilitando melhoria da qualidade do serviço.

$\mathrm{O}$ estudo constatou que as enfermeiras tinham prática satisfatória, realizando-a conforme a literatura. Entretanto, o desempenho dessas profissionais correspondeu parcialmente ao padrão de qualidade estabelecido pelo IMD.

Dentre as lacunas identificadas, algumas poderiam ser solucionadas com ações simples, de iniciativa local como por exemplo, a compra do ácido acético na concentração adequada, a utilização de materiais disponíveis (como sabão para a lavagem das mãos) e a imersão correta da lâmina no recipiente para fixação. Existem também aquelas lacunas que dependem de maior custo como é o caso da infraestrutura da unidade para disponibilizar lavatório no consultório.

A qualidade do exame de Papanicolaou depende 
da realização de todos os procedimentos estabelecidos pelo IMD. A inabilidade na realização de qualquer um destes compromete o exame em geral e afeta a segurança da cliente.

Neste contexto, constatou-se que o IMD é um instrumento eficaz para o controle de qualidade, pois oferece os parâmetros para monitoramento, supervisão e avaliação. Entretanto, por envolver o conceito de avaliação, esse instrumento deve ser passível de mudanças, adequando-se à realidade e recursos de cada região, sem deixar de seguir as diretrizes técnicas recomendadas.

\section{REFERÊNCIAS}

1. Ministério da Saúde (BR). Estimativa 2008: incidência de câncer no Brasil. [série online]. 2007 [Acesso 1 abr. 2008]. Disponível em: http://www.inca.gov.br/ estimativa/2008.

2. Secretaria da Saúde (CE). Saúde reprodutiva e sexual: um manual para a atenção primária e secundária (nível ambulatorial). Fortaleza: SES-CE; 2002.

3. Fernandes RAQ, Narchi NZ. Conhecimento de gestantes de uma comunidade carente sobre os exames de detecção precoce do câncer cévico-uterino e de mama. Rev Bras Canc. 2002 Jan/Mar;48(2):223-30.

4. Ministério da Saúde (BR). Instituto Nacional de Câncer. Ações de enfermagem para o controle do câncer: uma proposta de integração ensino - serviço. [série online] 2002 [Acesso 20 jul 2006]; p.148 Disponível em: http:// www.inca.gov.br/enfermagem/docs/cap3.pdf .

5. Nogueira RA, Moura ERF. Atuação de enfermeiras nas ações de prevenção do câncer cérvico-uterino. Ânima: Faculdade Integrada do Ceará. 2004 Jul/Dez;(6):37-43.

6. Ministério da Saúde (BR). Secretaria de Atenção à Saúde. Instituto Nacional de Câncer. Coordenação de Prevenção e Vigilância. Nomeclatura brasileira para laudos cervicais e condutas preconizadas: recomendações para profissionais de saúde. $2^{\mathrm{a}}$ ed. Rio de Janeiro: INCA; 2006.

7. Moura ERF, Leitão GCM. Importância do marco referencial e dos conceitos para um projeto de qualidade em saúde. Rev Eletrôn Enferm [periódico na internet]2000 Jul/Dez [acesso em 2006 Jul 15]2(2). Disponível em: www.fen.ufg.br/revista .

8. Peterlini O, Zagonel I. Explorando a avaliação: um instrumento básico para o gerenciamento do cuidado em saúde coletiva. Cogitare Enferm. 2003 Jul/ Dez;8(2):18-25.

9. Ceará. Secretaria da Saúde (CE). Metodologia de melhoria da qualidade da atenção à saúde: instrumento de melhoria do desempenho. $2^{\text {a }}$ ed. Fortaleza: SESA-CE; 2005.

10. Polit DF, Beck CT, Hungler BP. Fundamentos de pesquisa em enfermagem: métodos, avaliação e utilização. $5^{a}$ ed. Porto Alegre: Artmed; 2004.

11. Ministério da Saúde (BR). Conselho Nacional de Saúde. Resolução n. 196/96. Diretrizes e normas regulamentadoras de pesquisa envolvendo seres humanos. Diário Oficial da União, Brasília (DF), 16 out. 1996. Seção1, p. 21.082.

12. Ministério da Saúde (BR). Coordenação Nacional de DST e AIDS. Doenças sexualmente transmissíveis: manual de bolso. Brasília; 2000.

13. Zeferino L, Galvão L. Prevenção e controle do câncer de colo uterino: por que não acontece no Brasil? In: Galvão L, Díaz J, organizadoras. Saúde sexual e reprodutiva no Brasil: dilemas e desafios. São Paulo: Hucitec Population Council; 1999.p.346-60.

14. Swearingen PL, Howard CA. Atlas fotográfico de procedimentos de enfermagem. $3^{\mathrm{a}}$ ed. Porto Alegre: Artemed; 2001.

15. Tietjen L, Bossemeyer D, Mclntosh N. Prevenção de infecções: diretrizes para unidade de saúde com recursos limitados. Rascunho n. ${ }^{\circ}$ 2. Baltimore: JHPIEGO; 2005.

16. Smeltzer SC, Bare BG. Brunner/Suddarth: Tratado de enfermagem médico-cirúrgica. $9^{\mathrm{a}}$ ed. Rio de Janeiro: Guanabara Koogan; 2002.

17. Bickley LS, Szilagyi PG. Bases: Propedêutica médica. $8^{\mathrm{a}}$ ed. Rio de Janeiro: Guanabara Koogan; 2005.

18. Ministério da Saúde (BR). Prevenção do câncer do colo do útero: organizando a assistência (manual técnico). Brasilia; 2002.

19. Nettina SM. Brunner: prática de enfermagem. $7^{\mathrm{a}}$ ed. Rio de Janeiro: Guanabara Koogan; 2003. 